\title{
Electrostatic force microscopy studies of boron-doped diamond films
}

\author{
S. Gupta ${ }^{\text {a) }}$ \\ Department of Electrical and Computer Engineering, University of Missouri, \\ Columbia, Missouri 65211 \\ O.A. Williams \\ Institute for Materials Research, Universiteit Hasselt, BE-3590 Diepenbeek, Belgium \\ E. Bohannan \\ Department of Chemistry, University of Missouri, Rolla, Missouri 65409
}

(Received 12 December 2006; accepted 6 March 2007)

Much has been learned from electrochemical properties of boron-doped diamond (BDD) thin films synthesized using microwave plasma-assisted chemical vapor deposition about the factors influencing electrochemical activity, but some characteristics are still not entirely understood, such as its electrical conductivity in relation with microscale structure. Therefore, to effectively utilize these materials, understanding both the microscopic structure and physical (electrical, in particular) properties becomes indispensable. In addition to topography using atomic force microscopy, electrostatic force microscopy (EFM) in phase mode measuring the long-range electrostatic force gradients, helps to map the electrical conductivity heterogeneity of boron-doped micro-/nanocrystalline diamond surfaces. The mapping of electrical conductivity on boron doping and bias voltage is investigated.

Experimental results showed that the BDD films' surfaces were partially rougher with contrast of conductive regions (areas much less than $1 \mu \mathrm{m}^{2}$ in diameter), which were uniformly distributed. Usually, the EFM signal is a convolution of topography and electrostatic force, and the phase contrast was increased with boron doping. At the highest boron doping level, the conductive regions exhibited quasi-metallic electrical properties. Moreover, the presence of a "positive-negative-positive" phase shift along the line section indicates the presence of "insulating-conducting-insulating" phases, although qualitative. Furthermore, the electrical properties, such as capacitance and dielectric constants at operating frequency, were quantitatively evaluated through modeling the bias-dependent phase measurements using simple and approximate geometries. It was found that decreasing grain size (or increasing the boron concentration) lowers the dielectric constant, which is attributed to the change in the crystal field caused by surface bond contraction of the nanosized crystallites. These findings are complemented and validated with scanning electron microscopy, $\mathrm{x}$-ray diffraction, and "visible" Raman spectroscopy revealing their morphology, structure, and carbon-bonding configuration $\left(s p^{3}\right.$ versus $\left.s p^{2}\right)$, respectively. These results are significant in the development of electrochemical nano-/microelectrodes and diamond-based electronics.

\section{INTRODUCTION}

Diamond is a promising wide band-gap semiconductor material with a large potential offering excitement and interest due to its unique blend of superlative physical

\footnotetext{
a) Address all correspondence to this author.

e-mail: guptas@missouri.edu,sgup@ rocketmail.com

DOI: 10.1557/JMR.2007.0229
}

(electronic, optical, mechanical, and chemical) properties. ${ }^{1}$ Diamond thin films (DTF) in the form of poly-/ microcrystalline, nanocrystalline, and diamond-like carbon are attractive for numerous applications, such as in tribological coatings and cutting tools (extreme hardness), heat $\operatorname{sinks}^{2}$ (high thermal conductivity), optical windows (wide band gap, $5.45 \mathrm{eV}^{3}$ and wide spectral transparency), high-temperature and high-power electronics (breakdown voltage of $\sim 10^{7} \mathrm{~V} / \mathrm{cm}$ ), biosensors ${ }^{4}$ 
(chemical inertness), electrochemical electrodes (wide electrochemical window $)^{5,6}$ as a $p$ - $n$ junction diode or unipolar diamond-based electronics, ${ }^{7}$ radiation-hard electronics ${ }^{8}$ micro-/nanoelectromechanical systems, ${ }^{9}$ and vacuum microelectronics such as field-emission arrays, ${ }^{10}$ and therefore it is considered as an engineering material. The above-mentioned properties undoubtedly are unmatchable by any other material, and hence it is apparent that diamond is a material of choice in the twenty-first century.

Intense research efforts over the past two decades have yielded the technology to grow high-quality diamond thin films on nondiamond substrates, ${ }^{2,11}$ thus enabling some of the applications mentioned above. Nevertheless, to effectively utilize these materials for these applications, understanding their microscopic structure and physical (electrical and mechanical) properties becomes indispensable. A variety of diamond chemical vapor deposition (CVD) techniques currently available use different activation sources to create plasma (a soup of radicals and neutrals) include thermal (hot filament, flame), radiation [radio frequency (rf); microwave (MW)], electric fields [direct currect (dc) plasma], and others. ${ }^{12}$ Although these deposition techniques share some characteristics, each one has its own set of optimized processing conditions that can be used to produce films with different sets of structural and physical properties.

Doping in diamond to make it semiconducting (both $p$ - and $n$-type) is currently an intense subject of investigation. It is because electrically diamond is a wide bandgap semiconductor and limited by the fact that only p-type diamond is successful through boron doping. ${ }^{13}$ At room temperature, some of the valence band electrons are thermally promoted from the valence band maximum onto B-level, leaving free holes in the valence band, leading to the negatively charged acceptors and supporting the flow of current. Due to the relatively low dielectric constant of diamond, the Bohr radius for holes in diamond is small $(\sim 3 \AA)$, and the formation of an impurity band is also observed energetically for rather high boron concentrations $\left(>10^{19} \mathrm{~cm}^{-3}\right)$ located $\sim 0.37 \mathrm{eV}$, while the Mott insulator-metal transition is predicted to be $\sim 2 \times 10^{20} \mathrm{~B} \mathrm{~cm}^{-3} \cdot{ }^{14,15}$

Generally speaking, scanning-probe microscopy (SPM) is a simultaneous probe of topography and various physical forces with nanometer-scale resolution operated in multimode, enabling straightforward correlation of physical properties with the surface morphology of solid films. ${ }^{16,17}$ As the dimensions of the electronic devices become smaller (a few tens of nanometers), the ability to characterize electrical properties at microscopic scales gains even greater significance. ${ }^{18,19}$ Local electrostatic force measurements are particularly important to determine the electrical properties at the nanometer scale, including surface potential, charge distribution, dopant concentration, and dielectric constant in addition to the electrical characterization of integrated circuits and nanometer-scale devices. ${ }^{18}$ Electrostatic force microscopy (EFM), a noncontact or tapping SPM technique, allows fast two-dimensional voltage contrast measurements which can be acquired either in scanning Kelvin probe microscopy (SKPM) or EFM-phase mode. Note that EFM can also be operated in amplitude and frequency modulation modes. Although SKPM provides a direct surface potential measurement, its spatial resolution is relatively poor due to long-range Coulomb forces, resulting in smearing of the actual potential distributions. On the other hand, the EFM-phase method is based on the measurement of the electric force gradient rather than that of force itself, thus producing a higher-resolution measurement of the surface potential. Recently, spatial resolution better than $20 \mathrm{~nm}$ has been achieved using EFM-phase method as compared to $\sim 100 \mathrm{~nm}$ in SKPM. ${ }^{16,20}$ SKPM has been used to measure the potential distribution in laser devices, semiconductor polymer field-effect transistor (FET) devices, and the electronic domain structure in a doped monolayer of a conducting polymer, whereas the EFM-phase method has been used to characterize the electrical properties of carbon nanotubes, ${ }^{18} \mathrm{DNA}^{21}$ and electrical conductivity heterogeneity in B-doped diamond films. ${ }^{22}$ The latter has also been used to (i) image small droplets situated upon a substrate on nanometer-length scales, (ii) study the charge mobility on ionic surfaces, (iii) map the compositional pattern of buried organic interfaces, and (iv) quantify the characteristics of charged (or uncharged) surface sites. Because the phase shift is related to the surface potential rather indirectly, quantification of the EFM-phase measurement is needed, which is an ongoing research activity.

In principle, EFM-phase method is a two-pass technique measuring topography during the first pass and another selected property of the sample using topographical information to track the tip at a constant distance above the surface during the second pass. This minimizes the interference of two kinds of data: topographical and electrostatic. This technique requires use of a conducting probe, which is usually fabricated by applying special conductive coatings on traditional tips. The coatings are deposited on both sides of the cantilever to prevent bending. The probe should have a force constant small enough to have good sensitivity to the electrostatic forces. When the second pass is used for measurement of electric-field gradient and distribution above the surface, the technique is referred to as EFM. The phase of the alternating current (ac) wave form from the photo diode assembly is compared to the drive signal applied to the tapping piezo on the cantilever holder. Monitoring the phase of the cantilever's vibration allows the microscope to record electrical changes on the 
sample during scanning. For the EFM experiments, the conductive tip causes an attractive or repulsive force to exist between the tip and the sample. The force causes the resonant frequency to change, which in turn causes the phase of the cantilever vibration to change. A schematic of the EFM-phase operation principle is shown in Fig. 1.

The use of boron-doped diamond (BDD) films in electronics and specifically as microelectrodes in electrochemistry is an exciting field of research for this technologically important material. In the present study, the electrical heterogeneity of high-quality BDD film surfaces with different boron doping levels is investigated using the EFM-phase technique. We showed through analytical calculations and fitting the experimental data well that the EFM-phase shift which is equivalent to the change in resonant frequency, either lower or higher, is related to the attractive (conducting) and repulsive (insulating) forces, thus helping to determine the capacitance and dielectric constant subsequently. ${ }^{23}$ Dielectric polarization, which determines the dielectric constant of a material, is expressed as the sum of three factors: dipolar, ionic, and electronic polarizations. Because the contribution from electronic polarization is dominant at high frequencies, dielectric materials with a low electronic polarization are necessary. The dielectric constant of diamond does not change over a large frequency range because the dipolar and ionic polarizations are negligible, in addition to the localized electronic or atomic orbitals of the $s p^{3}$-bonded carbon $\left(s p^{3} \mathrm{C}\right)$ suppressing the electronic polarization. Due to the high density, $3.51 \mathrm{~g} / \mathrm{cm}^{3}$, the relative dielectric constant $\left(\epsilon_{\mathrm{r}}\right)$ of diamond is $5.68 .^{24}$ Following the procedure described in Ref. 24, the experimental results were obtained under various biases. The results show that the film surfaces were nonconducting, with contrast of conducting regions randomly distributed over the surface and the contrast increasing with bias and boron concentration. These measurements are complemented with and validated using scanning electron microscopy (SEM), x-ray diffraction (XRD), and Raman spectroscopy (RS) tools to reveal their microscopic structure and carbon-bonding variations. Moreover, detailed microscale structural studies were able to demonstrate that the carbon-bonding configuration $\left(s p^{2}\right.$ versus $s p^{3}$ hybridization) and surface chemical termination in both the undoped and doped diamond have a strong influence on nanoscale electrical properties.

\section{EXPERIMENTAL PROCEDURES}

Thin films of BDD films were synthesized using a commercial ASTeX (Woburn, MA) 5-kW microwave plasma-assisted chemical vapor deposition technique onto conductive $n$-type $\mathrm{Si}(001)$ substrates $(0.05 \mathrm{~cm}$ thick, $1 \mathrm{~cm}^{2}$ in area, $10^{-3} \Omega$-cm; Virginia Semiconductor, Inc., Fredricksburg, VA), described elsewhere. ${ }^{25,26}$ Briefly, silicon wafers were ultrasonically rinsed in ethanol, followed by seeding in solutions of ethanol and nanodiamond powder with a grain size of $3-5 \mathrm{~nm}$. However, it should be noted that these particles aggregate to sizes $\sim 100 \mathrm{~nm}$. Embedded diamond powder and the scratching residues introduced by ultrasonication serve as initial nucleation sites. These films were deposited at 2000-3000 W, using a $\mathrm{CH}_{4} / \mathrm{H}_{2} /$ trimethylborate [TMB; $\mathrm{B}\left(\mathrm{OCH}_{3}\right)_{3}$ for boron] source gas mixtures, with total chamber pressure of 30-40 Torr at the substrate temperature $\sim 750{ }^{\circ} \mathrm{C}$ (estimated via an optical pyrometer), and a growth time of 2-3 h. At the end of the deposition period, the $\mathrm{CH}_{4}$ and TMB gas flows were stopped and the films

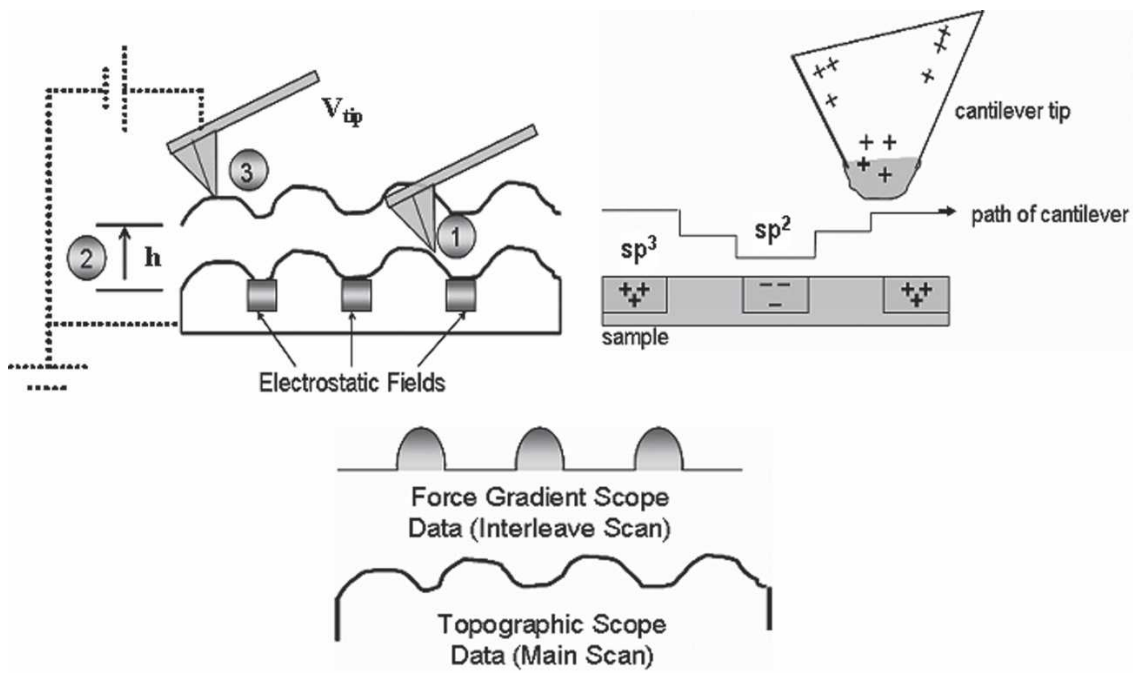

FIG. 1. Schematic of experimental set-up for EFM measurements, with the principle of the LiftMode operation: (1) cantilever measures surface topographical features on first (main) scan; (2) cantilever ascends to lift scan height $(h)$; (3) cantilever follows stored surface topography at the lift height above sample while responding to electric influences or electrostatic force gradients on second (interleave) scan. 
remained exposed to $\mathrm{H}_{2}$ plasma at 2000 and 40 Torr for an additional $10 \mathrm{~min}$. The plasma power and pressure were then slowly reduced over a 5-min period to cool the samples to a temperature $<400{ }^{\circ} \mathrm{C}$, in the presence of atomic hydrogen. The plasma power was then turned off, and the films were cooled to room temperature under a flow of $\mathrm{H}_{2}$. This post-growth annealing in atomic hydrogen served to gasify any adventitious nondiamond $s p^{2}$ carbon impurity, to minimize dangling bonds, and to fully hydrogenate the surface. The boron concentrations in gas phase and thickness were $500 \mathrm{ppm}, 1 \mu \mathrm{m}$ (4$1110 \mathrm{~A}$; A), $800 \mathrm{ppm}, 200 \mathrm{~nm}$ (5-0105B; B), $800 \mathrm{ppm}, 1 \mu \mathrm{m}$ (5-0105B; C), and $2000 \mathrm{ppm}$ (5-0103A; D), respectively, followed by the samples' identification (ID) in parentheses. The room-temperature electrical conductivity and carrier concentration of these films are approximately $0.1-10 \mathrm{~S} \mathrm{~cm}^{-1}$ and $\geqslant 10^{19}-10^{20} \mathrm{~cm}^{-3}$ determined using Hall probe measurements, respectively. ${ }^{25}$

The film thickness was $\sim 0.5 \mu \mathrm{m}$ as measured mechanically using a Tencor surface profilometer (KLATencor, San Jose, CA). As-deposited diamond samples were characterized by SEM using a JEOL (Tokyo, Japan) model 3400 instrument to reveal their morphologies. Samples were also characterized using atomic force microscopy (AFM; dimension 3100 NS-IIIA instrument; Veeco Instruments, Santa Barbara, CA), XRD, and RS. $\theta-2 \theta$ XRD scans were measured with a Scintag 2000 XDS instrument (Scintag, Cupertino, CA) using $\mathrm{Cu} \mathrm{K}_{\alpha}$ $(1.5405 \AA) \mathrm{x}$-ray source. XRD is used to determine the preferential crystallite orientation of the films. RS analyses were carried out using 514.5-nm (2.41-eV) excitation from an $\mathrm{Ar}^{+}$-laser with a ISA J-Y TRIAX 320 spectrometer (ISA, Inc., Edison, NJ) in backscattering geometry to analyze the structural bonding in the films. All spectra were measured with a beam spot size of $\sim 2 \mu \mathrm{m}$ with minimum power $\left(\sim 10 \mathrm{~kW} / \mathrm{cm}^{2}\right)$ to avoid thermal degradation. All the XRD and Raman spectra were fitted using Jandel Scientific PeakFit software (ver. 4.0; Jandel Scientific, Corte Madera, CA) based on the MarquardtLevenberg method. ${ }^{27}$

For the EFM-phase measurements, we have used a Veeco Instruments (dimension 3100 NS-IIIA) AFM. The substrate for all experiments is degenerately doped $p$ type $\mathrm{Si}$. A $200-\mathrm{nm} \mathrm{SiO}_{2}$ layer on top of a $p$-type degenerately doped $\mathrm{Si}$ wafer was used to calibrate the equipment to determine the background phase lag. ${ }^{19}$ In the EFM dual-pass technique, the tip acquires a topography profile in tapping mode in the first line scan. In the second line scan (see Fig. 1), i.e., interleave mode, the tip travels at a defined height $(h)$ above the surface. A dc voltage is applied to the tip, and the cantilever is mechanically driven at its resonant frequency $\left(\omega_{0}=79 \mathrm{kHz}\right)$. The EFM-phase image records the phase of the cantilever oscillation as a function of tip position. The images were taken using a $n^{+}$-Si tip coated with metal, known as an micro-etched silicone probe (MESP) tip, having radius of curvature, $R_{\text {tip }}$, of $25 \mathrm{~nm}$, quality factor $Q=300$, and spring constant $k=2.8 \mathrm{~N} / \mathrm{m}$. The phase signal refers to the timing of the mechanical vibration of the cantilever relative to the movement of the cantilever mount. We measured both in positive and negative voltages at ambient conditions, and the results were similar except that the contrasts were reversed.

\section{RESULTS AND DISCUSSION}

\section{A. Materials characterizations}

Scanning electron micrographs were obtained on all of the samples to reveal morphology, as shown in Fig. 2. For comparison, an undoped diamond film SEM image is also shown. All of the SEM micrographs showed that the films are micro-/nanocrystalline with uniform surface coverage with no visible cracks, pinholes, or voids, except that the undoped film appears to be somewhat polycrystalline. The SEM micrographs also showed that the films are composed of well-faceted diamond crystallites (with triangular growth habit or $\langle 111\rangle$ facets) for undoped that starts to turn finely grained nonfaceted texture on doping with feature sizes on the order of $<100 \mathrm{~nm}$, which decreased further with increasing boron concentration in gas phase. The decreasing grain size and smoother surfaces with increasing boron concentration are attributed to the high rates of renucleation or secondary nucleation, which is heterogeneous, ${ }^{28}$ and they are analogous to nitrogen and sulfur incorporations. ${ }^{29}$ While SEM is qualitative, AFM was used to quantitatively assess the grain size and surface roughness. Figure 3 shows the threedimensional (3D) AFM images and the corresponding analyses confirming the qualitative observations made from SEM images. The AFM analyses indicated that the average grain size varied from 0.5 to $0.2 \mu \mathrm{m}$ for the undoped and boron-doped diamond films. Variations in the root mean square $(\mathrm{rms})$ surface roughness $\left(\sigma_{\mathrm{rms}}\right)$ values ranged between 180 and $100 \mathrm{~nm}$ and 40 and $22 \mathrm{~nm}$ for the undoped and boron-doped diamond films, respectively. Note that the doping or impurity additions reduce the surface roughness in general. When compared to $\mathrm{N}$ addition, the thermodynamic calculations suggested that they induce a transition from faceted to unfaceted morphology by activated species (possibly HCB and HCS analogous to $\mathrm{HCN}){ }^{29}$

Figure 4 depicts the XRD diffractogram for the representative samples as mentioned above, showing the characteristic diamond (111) peak at $2 \theta=44^{\circ}$ in addition to other diamond peaks (220 and 311), which clearly indicates that all of the diamond films are polycrystalline in nature. Qualitatively, the full width at half-maximum (FWHM) of the characteristic XRD diamond peak becomes broader with increasing boron incorporation. 


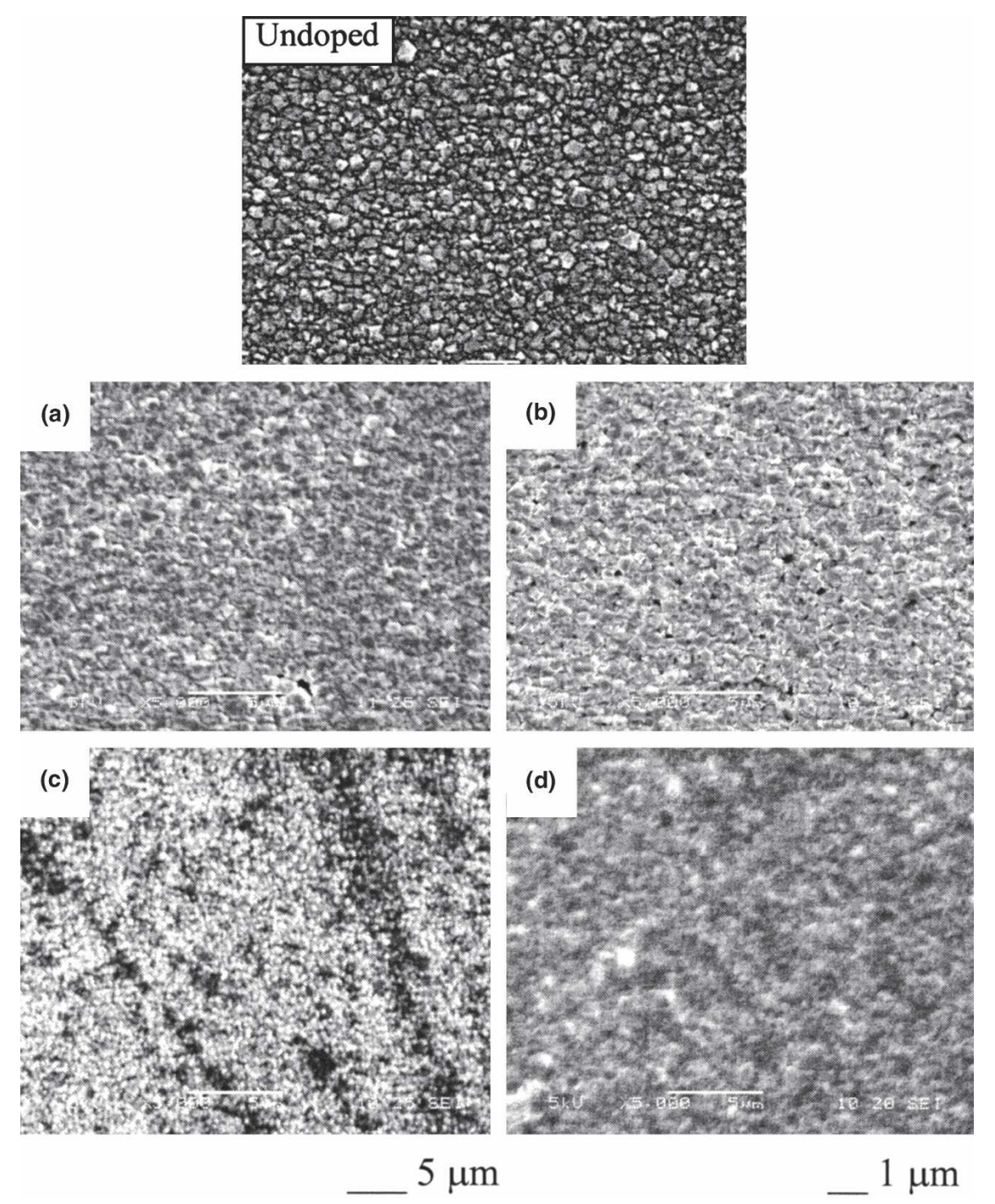

FIG. 2. Scanning electron micrographs of the heavily B-doped diamond films as a function of boron concentration. Boron concentration in gas phase and film thicknesses are also provided.

Also, it shifts toward higher $2 \theta$ values, indicating development of compressive stress in the films. The corresponding crystallite sizes were estimated using DebyeScherrer method: $\left(L_{h k l}=K \lambda / b \cos \theta\right)$, where $K \approx 1$ (Debye-Scherrer constant), $\lambda=1.5405 \AA\left(\mathrm{Cu} \mathrm{K}_{\alpha}\right)$, and $b$ is the FWHM of $<111>$ peak. ${ }^{30}$ The values are in good agreement with the ones estimated using AFM grain-size analysis, rather qualitatively. The lattice parameter $(a)$, on the other hand, varied from 0.3560 to $0.3570 \mathrm{~nm}$ with increasing boron concentration.

RS has emerged as one of the most frequently used analytical tools to characterize carbon-based materials, in general, ${ }^{31}$ and of diamond thin films in particular. ${ }^{32} \mathrm{Be}-$ cause Raman analyses can detect changes in behavior of $\mathrm{C}-\mathrm{C}$ bonds, probing microstructural variation due to any treatment is much more informative and quantitative. Visible RS ( $\lambda_{\mathrm{L}}=514.5 \mathrm{~nm}$ or $\left.E_{\mathrm{L}}=2.41 \mathrm{eV}\right)$ is used to assess the crystalline quality and to probe the changes in the structural bonding configurations in BDD films. In addition, because Raman scattering depends upon the electron-phonon interactions, it is sensitive to local environment. Given the importance of doping in diamond, it appears that no simple signature can be used to assess doping levels. Instead, the signatures are subtle, involving interactions between lattice vibrations and electronic continuum of states induced by the dopants. Figure 5 shows the Raman spectra of boron-doped diamond films as a function of boron concentration in the gas phase provided by the samples' ID. The RS in terms of peak position, peak widths, relative intensities, or integrated area for diamond films is quite sensitive to the bulk crystal structure, the boron-doping level, the internal stress (intrinsic and thermal), the defect density, and/or the presence of $s p^{2}$-bonded carbon impurity. ${ }^{33-40}$

Qualitatively, RS analysis revealed the one-phonon zone center line at $1332 \mathrm{~cm}^{-1}$, which is the most intense 

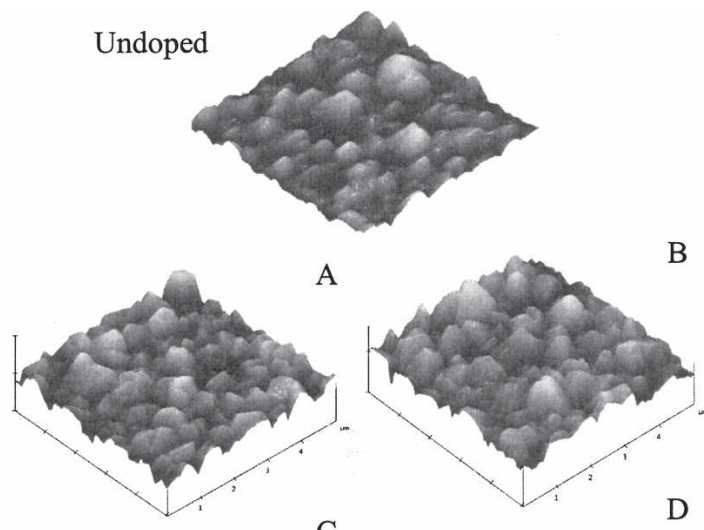

C

$\mathrm{D}$
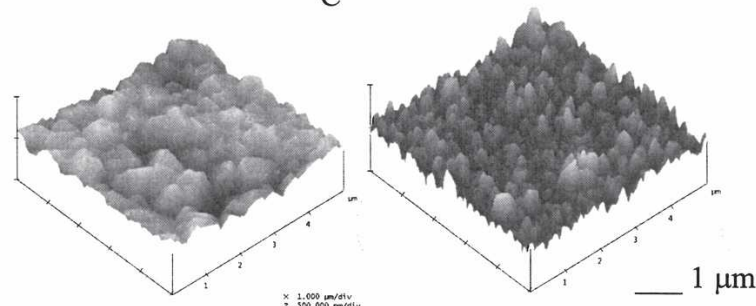

$1 \mu \mathrm{m}$

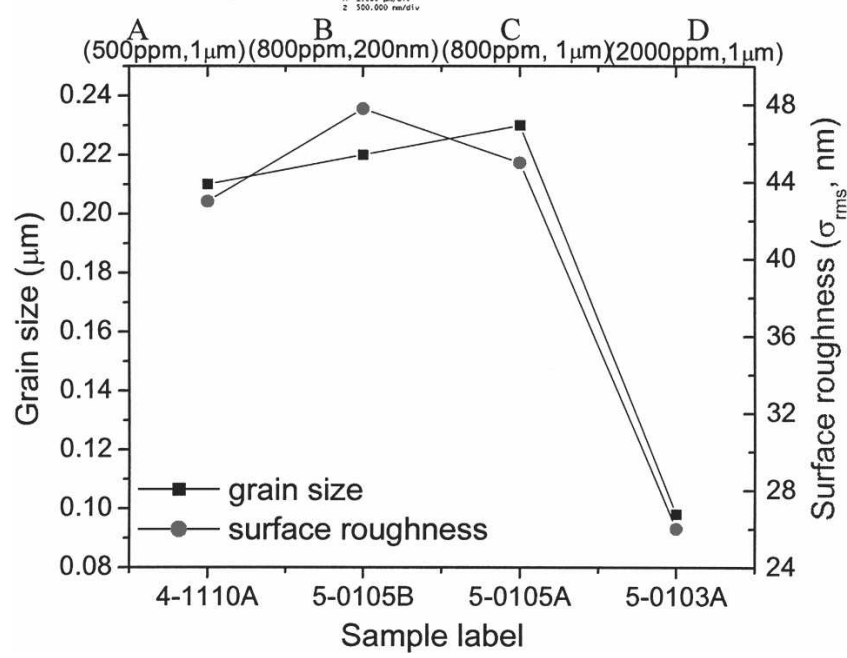

FIG. 3. Three-dimensional AFM images of the heavily B-doped diamond films as shown in Fig. 1 and the corresponding analyses in terms of average grain size analyses and rms surface roughness.

and narrow for the lowest B doping. Although the characteristic diamond peak position did not change significantly, the line shape did become asymmetric for the highest B-doping level. This is known as the Fano effect, which is induced by quantum-mechanical interference between the discrete phonon states and electronic continuum. ${ }^{41-43}$ The strongest effect appears to occur when the doping level is $\sim 2 \times 10^{20} \mathrm{~cm}^{-3}$, which corresponds to the onset of quasi-metallic nature of the films i.e., near-zero activation energy and it is for samples higher than $1000 \mathrm{ppm}$ of TMB in present case. It implies that this occurs only when the density of dopants is sufficiently high that they form a continuous impurity band. Additionally, the Raman peak due to silicon substrate decreases (data not shown) with increasing boron concentration; as the doped material becomes opaque at the excitation wavelength, new peaks emerge near $1230 \mathrm{~cm}^{-1}$ for these films. Minimal scattering intensity is seen in the $1500-1600 \mathrm{~cm}^{-1}$ region, which is associated with the presence of nondiamond or $s p^{2}$-bonded carbon $\left(s p^{2} \mathrm{C}\right)$. These spectral characteristics are consistent with heavily boron-doped films. ${ }^{32,41-43}$ Moreover, we also observed that the small $s p^{3}$-bonded diamond grains are connected to one another by grain boundary with $s p^{2}$ bonding carbon configuration. The peaks seen at $\sim 1150,1333$, 1470 , and $1550 \mathrm{~cm}^{-1}$ are relatively broad. The peak width (FWHM; $\Gamma$ ) for the diamond line is much broader than that for the other larger grained diamond films, i.e., 70 versus $15 \mathrm{~cm}^{-1}$. There are two possible explanations for the line broadening, both related to the nominal grain size. One possibility is the well-established confinement model. ${ }^{38}$ This model states that the smaller the grain size, the larger the range of phonon modes (with different $\mathbf{q}$ vectors and energies) that is allowed to participate in the Raman process. Hence, the linewidth results from the spread in phonon energy. Another and more likely explanation is phonon scattering by impurities and defects (i.e., grain boundaries). ${ }^{38}$ The scattering event shortens the lifetime of the phonons, $\tau \propto 1 / \Gamma$, and thus broadens the characteristic diamond band in Raman spectra.

The peak at $1150 \mathrm{~cm}^{-1}$ is often used as a signature for high-quality nanocrystalline diamond. ${ }^{36}$ Prawer and coworkers $^{44}$ through the study of clean nanocrystalline diamond particles $(\sim 5 \mathrm{~nm}$ in diameter) have attributed this peak to a surface phonon mode of diamond. On the other hand, Ferrari and Robertson and others have made arguments for this peak being associated with $s p^{2}$-bonded carbon, specifically trans-polyacetylene segments at grain boundaries. ${ }^{45-47}$ Their assignment of $s p^{2}$ rather than $s p^{3}$-bonded carbon, which has been often proposed, ${ }^{36}$ is based on the observations that the peak position changes with excitation energy, the peak intensity decreases with increasing excitation energy, and the peak is always accompanied by another peak at $\sim 1450 \mathrm{~cm}^{-1}$. We therefore tentatively assign the peaks at 1470 and $1550 \mathrm{~cm}^{-1}$ to disordered $s p^{2}$-bonded carbon in the grain boundaries. It is important to note that the disordered carbon is confined to the grain boundaries of the micro-/ nanocrystalline films, producing a network of 3- $\left(s p^{2}\right)$ and fourfold $\left(s p^{3}\right)$ coordinated carbon atoms. ${ }^{29,48}$

\section{B. EFM investigations}

High-quality BDD films are studied using the EFMphase technique to map the topography in conjunction with the electrostatic force gradient providing electrical conductivity heterogeneity. The dependence of electrical conductivity on boron doping level and bias voltage polarity is studied. First, we address the EFM calibration method followed by the investigations on BDD films and 


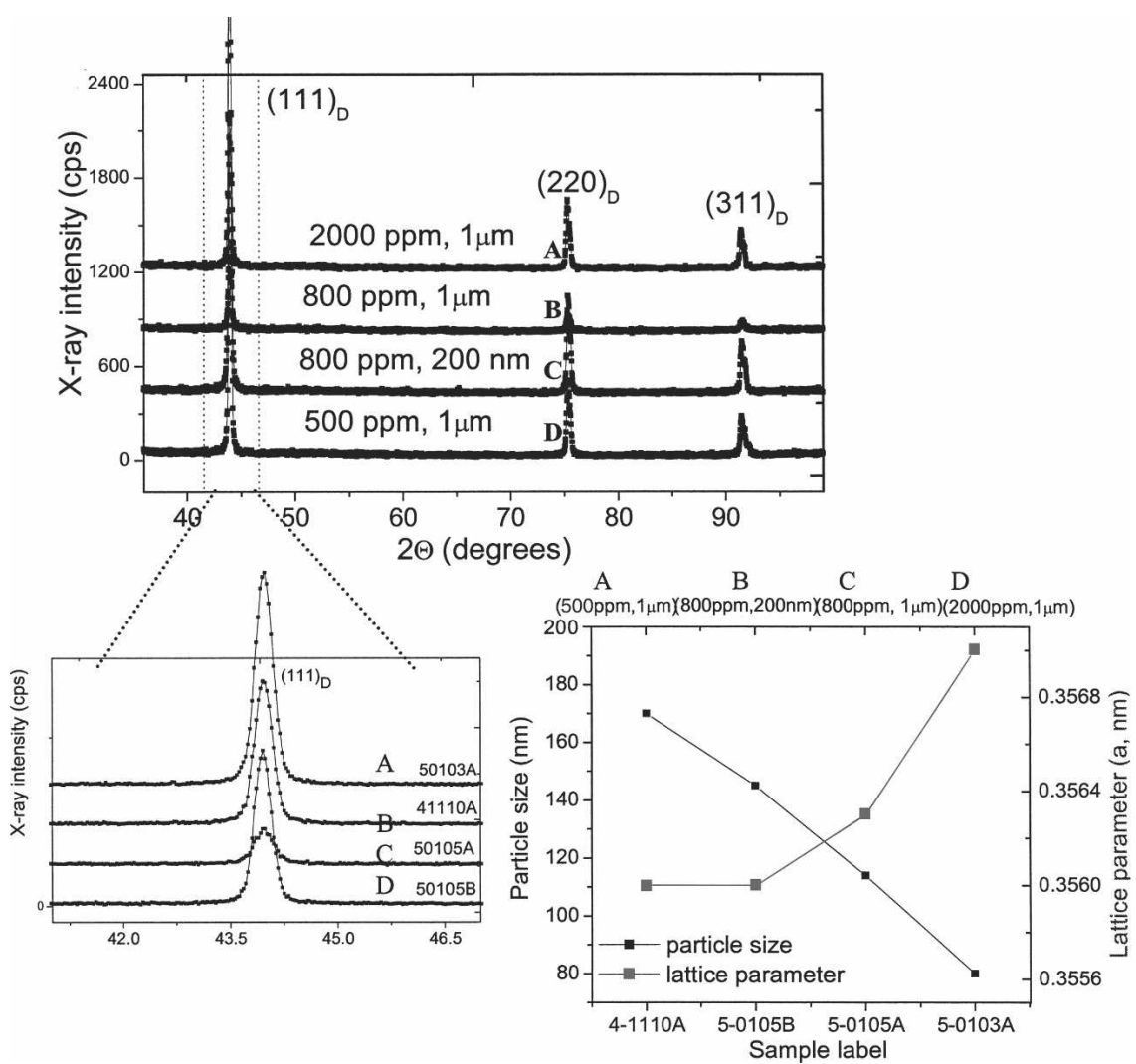

FIG. 4. X-ray diffractograms of the heavily B-doped diamond films as shown in Fig. 1 displaying characteristic diamond peak, zoomed-in version, and analyses in terms of crystallite size and lattice parameter $(a)$.

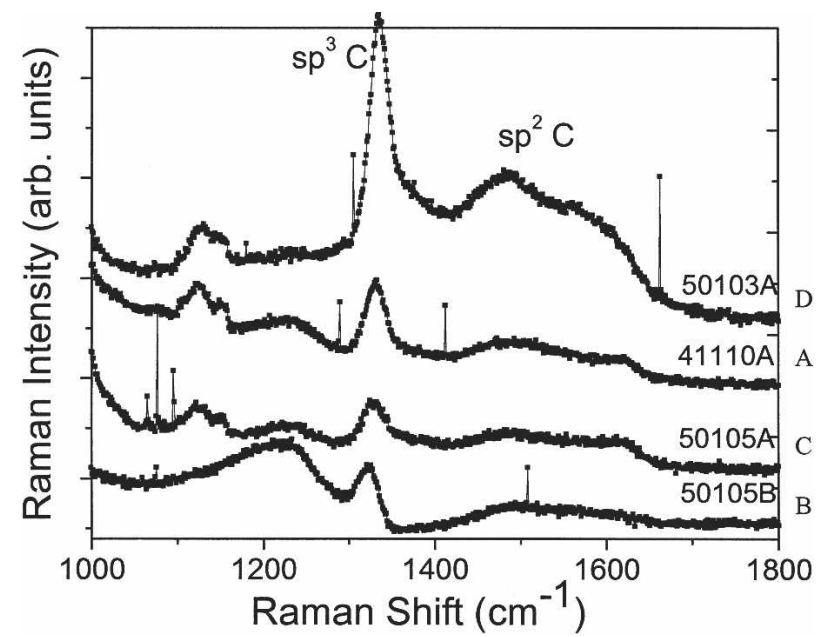

FIG. 5. Visible Raman spectra of the heavily B-doped diamond films as shown in Fig. 1 displaying significant Raman signatures, including $s p^{3}$ - and $s p^{2}$-bonded carbon.

corresponding modeling to determine electrical properties, such as capacitance and relative dielectric constant.

\section{Calibration}

Quantitative calibration of EFM-phase measurement involves relating the phase shift directly to the local sur- face potential, which needs to be performed on a flat sample whose surface potential is known. For instance, a thermal evaporated gold film on $\mathrm{SiO}_{2}$ may serve this purpose. The phase shift was recorded as a function of applied voltage and tip lift height $(h)$. Figure 6 is a plot of phase shifts at different biases between the film and the probe or tip, measured by EFM-phase, with a fixed lift height of $50 \mathrm{~nm}$. The data obtained over a $2-\mu \mathrm{m}$ scan line were averaged along the scan line, and the trace taken at $0 \mathrm{~V}$ bias was subtracted from them. The parabolic-phase potential relationship is valid at low biases, as described by the equation ${ }^{19}$ :

$$
\Delta \phi=-\arcsin \left[\frac{Q}{2 k} \frac{d^{2} C}{d z^{2}}\left(\Delta V_{\text {tip }}\right)^{2}\right],
$$

which relates the phase or frequency shift $(\Delta \phi)$ and the potential difference $\left(\Delta V_{\text {tip }}\right)$. The resolution of the EFMphase technique is determined by the lateral distribution of $d^{2} C / d z^{2}$. This phase shift will always be negative, because $d^{2} C / d z^{2}$ is positive. At the same time, the electrostatic force between the tip and surface is attractive because $d C / d z$ (the capacitance gradient) is negative. Notice that the equation is an approximation by assuming that $\omega \simeq \omega_{0}$. This equation may not be precise for high voltages, i.e., $>10 V_{\mathrm{dc}}$, due to increased electrostatic interaction, as observed in Fig. 6. $k$ and $Q$ are constant for 


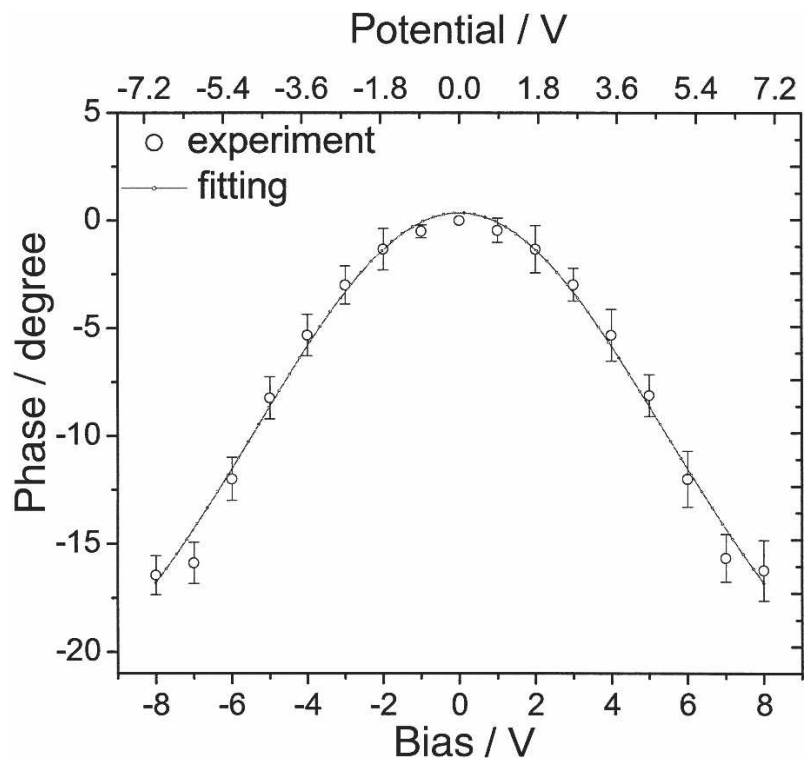

FIG. 6. EFM phase shift at different bias on the metal tip with respect to the heavily B-doped diamond films. Lift height is $50 \mathrm{~nm}$. The solid curve is a least-square fit to the data, using the equation $\Phi=-\arcsin \left[0.0075(V-0.389)^{2}\right]+0.105$. The corresponding potential calculated from the equation $\Phi=-\arcsin \left[0.0082(V-0.123)^{2}\right]+$ 0.162 is also shown (top abscissa).

a certain tip and $d^{2} C / d z^{2}$ is constant when the tip-sample separation is fixed and the tip and sample are metallic. If the tip or sample is a semiconductor, $d^{2} C / d z^{2}$ varies with bias $V_{\text {tip }}$ as discussed. To begin with, we assume that $d^{2} C / d z^{2}$ does not vary with bias. The data were fitted in the range of -8 to $+8 \mathrm{~V}$ using least-squares method to give the solid curve in the Fig. 6. The fit function used was

$$
\phi=-\arcsin \left[A\left(V_{\text {tip }}-V_{0}\right)^{2}\right]+B,
$$

where $A=0.0075 \pm 0.0001 V_{\text {tip }}{ }^{-2}$ represents the constant parameter $(Q / 2 k) d^{2} C / d z^{2}$, and $B=0.105^{\circ} \pm 0.009^{\circ}$ is an offset to $0^{\circ}$ when $V=V_{0}\left(V_{0}=0.389 \pm 0.012 V\right)$. It represents bias offset that arises mainly from the work function difference between the Si probe and the calibrating gold film. ${ }^{23,49,50}$ The lift height $(h)$ is $50 \mathrm{~nm}$. The corresponding potential calculated from the equation

$$
\Phi=-\arcsin \left[0.0082(V-0.123)^{2}\right]+0.162,
$$

is also shown (top abscissa in Fig. 6).

Equation (1) describes well the observations for a large phase shift of $25^{\circ}$, in both positive and negative biases. This might be related to the high-quality factor $Q$ of the probe which, with a small frequency shift, can result in a considerable phase shift. The slight difference in the phase shift due to positive and negative bias is related to the semiconducting nature of the tip. The depletion width in the tip is a function of the bias strength and bias polarization/polarity. Therefore, the capacitance and its gradient (both first and second order) with applied volt- age vary with the biases. For instance, at the same bias strength, the tip-sample capacitance gradient is smaller in the accumulation state (positive bias) than in the depletion state (negative bias). From these considerations, it appears that the electrostatic force and phase shift are larger in reverse bias than those in the forward bias. Hence accumulation state (positive bias) is beneficial to the resolution because the carriers are accumulated in the apex region of the tip.

\section{Application to BDD films}

DTF can possess electronic properties ranging from those of an insulator to semiconductor when doped from low to moderate levels and to those of a semimetal at higher doping levels. ${ }^{51,52}$ Figures 7(a)-7(d) show EFMphase images (right), corresponding topography (left) in $5 \times 5 \mu \mathrm{m}^{2}$ region and line scans for BDD films as a function of positive dc voltage $\left(V_{\mathrm{dc}}\right)$ applied to the tip with respect to substrate/films and with increasing boron concentration. EFM phase is a type of scanning-probe microscopy that measures electric-field gradients near the surface of a sample using a sharp conductive tip described above. We noted a variety of important observations induced by tip biasing.

Qualitatively, the topography images show only surface roughness and impressions of crystallites, which become sharper in EFM-phase images with contrasting regions depending upon electrical nature in the region. Notice that the EFM-phase image was featureless at $0 \mathrm{~V}$, as desired [Fig. 7(a)]. The EFM-phase images show that the samples are insulating over much of the probed area, with most of the surface recording almost zero phase shift. While the phase shift increased with increasing bias voltage [see line scans at $8 \mathrm{~V}$; Fig. 7(d)]. The areas of conductivity are on the order of submicron diameter, and they are randomly distributed over the diamond surfaces. Some of the conductive regions appear to be clustered together, whereas others appear to be completely isolated. Alternatively, the boron-doped diamond surfaces seem to be spatially inhomogeneous. Furthermore, there seems to be a correlation between topography and electrical conductivity or electric force gradient. The areas of high conductivity may be associated either with the grain boundaries separating the diamond crystallites and they tend to accumulate $s p^{2}$-bonded carbon $\left(s p^{2} \mathrm{C}\right)$ or the presence of boron. Because electrical contact was made from the bottom $p$-Si substrate, this indicates that conduction is possible along only certain pathways through the substrate to the diamond film surface. Thus, areas of high electrical conductivity correspond to sites where surface boron dopant sites can establish electrical contact to the substrate through the film. The distribution of dopant on the surface could be greater than that detected by EFM-phase measurements, but many boron sites may be electrically inactive due to a lack of conductive pathways 
within the film or passivation with $\mathrm{H}$ and adventitious $\mathrm{N} .{ }^{25}$ Landrass and Ravi demonstrated the influential role of hydrogen in controlling or enhancing the electrical resistivity through defect passivation. ${ }^{53}$ The dopant is likely to be nonuniformly distributed within the diamond crystallites, leading to electrical heterogeneity but relatively uniform on the diamond surfaces. Boron doping is said to be heaviest on $\{111\}$ crystal faces $^{54}$ as well as being concentrated in grain boundaries. A high electrical force gradient is observed in the intercrystallite regions, suggesting that boron is present in high concentrations at those sites or that conduction through the film is facilitated by the presence of $s p^{2}$ inclusions/defects. The density of conducting areas increased with doping level, thus confirming that these areas are associated with boron sites.

Additionally, the contrast variation is much more prominent for EFM phase in contrast to surface potential measured using surface Kelvin microscopy (SKM) (not shown). However, the reasons are not understood. It may originate from potential-dependent tunneling between grain boundaries and boron-doped diamond crystallites, similar to the collective charge transport in a twodimensional array of metadots and polymer chains separated by tunnel barriers. ${ }^{55}$ The higher lateral resolution achievable with EFM-phase response is faster than its amplitude response to changes in tip-sample interactions and less susceptible to height variation on the sample surface. The key issue is to quantify the EFM-phase measurement to determine the local surface potential, capacitance and dielectric constant through detailed analyses, possibly through modeling.

\section{Modeling of tip-sample capacitance}

Unfortunately, it is known that quantitative interpretation of the AFM signal is rather difficult because the signal depends invariably in a complicated fashion upon the tip shape and geometry. Here, we made an attempt to model the EFM-phase signals shown in Fig. 7. It can be explained by considering a cantilever of resonant frequency $\omega_{0}$ and spring constant $k$. For a cantilever driven in air (damping coefficient; $\gamma$ ) at frequency $\omega$, the phase

FIG. 7. (a-d) EFM-phase images of the heavily B-doped diamond samples as a function of tip bias with increasing boron concentration similar to shown in Fig. 3. Corresponding two-dimensional topography images are also shown on the left, measured simultaneously. Both of these images are measured in tapping mode and electric force microscopy with $V_{\text {tip }}=0,2,4,6$, and $8 \mathrm{~V}$ peak to peak at a frequency of $79 \mathrm{kHz}$ and tip-sample separation $(h)$ of $50 \mathrm{~nm}$. Images in the reverse bias are not shown because they are similar to the positive bias except that the contrasts are reversed. The line scan showing the phase versus tip position is shown for the highest tip bias of $8 \mathrm{~V}$ for all of the samples. Note the degree of contrast between conductive regions (lighter areas) and insulating regions (darker areas) in EFM-phase images. (a)
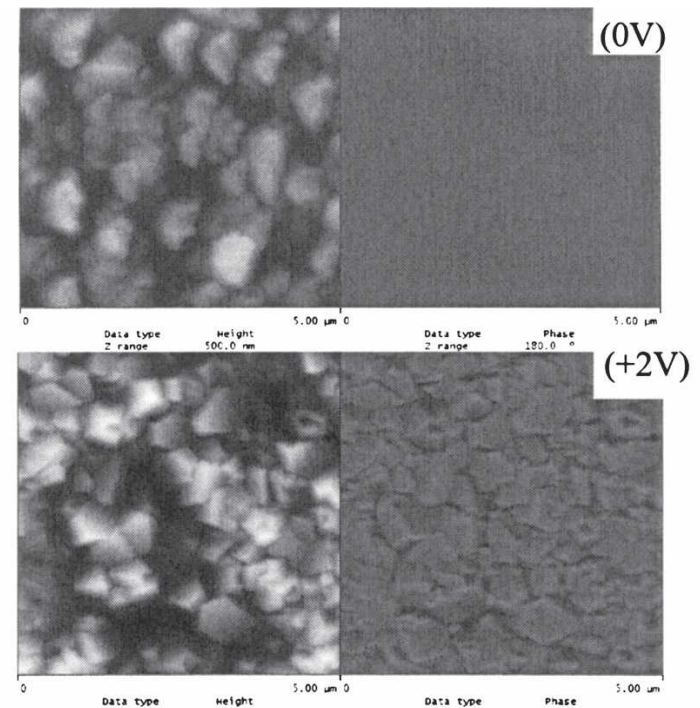

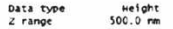

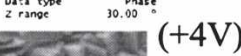
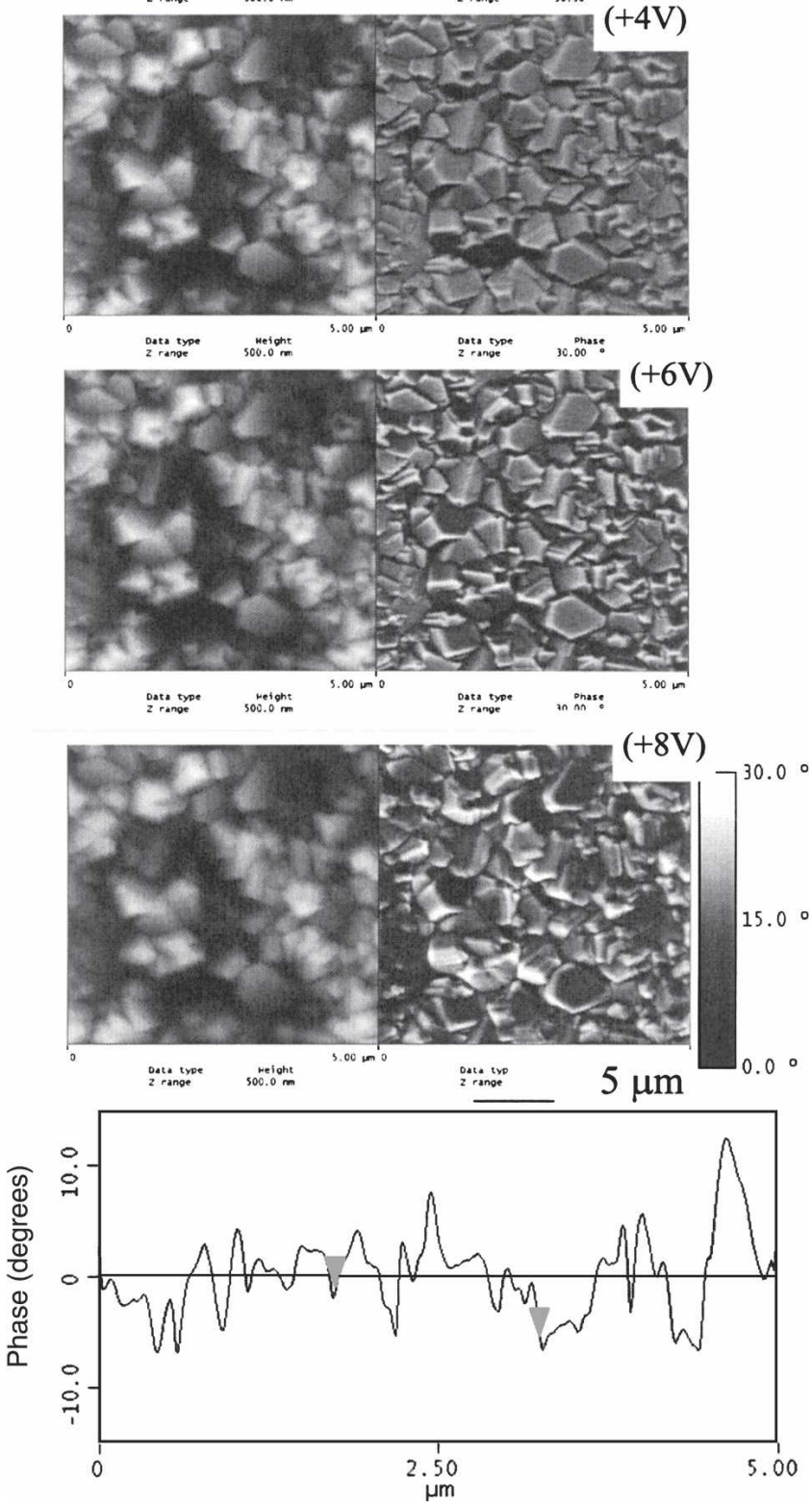
(b)
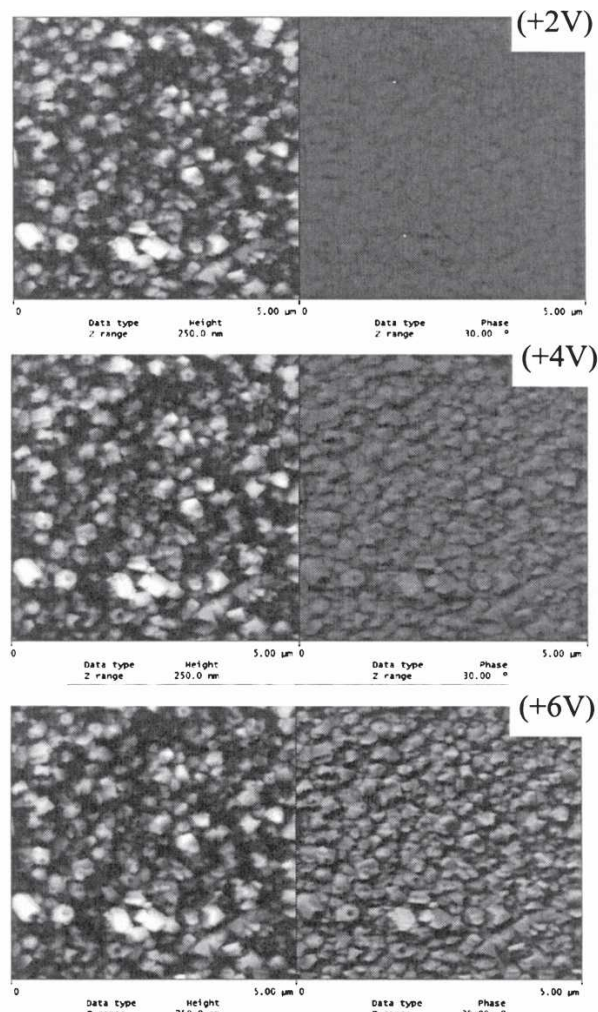

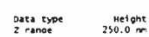

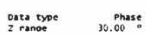
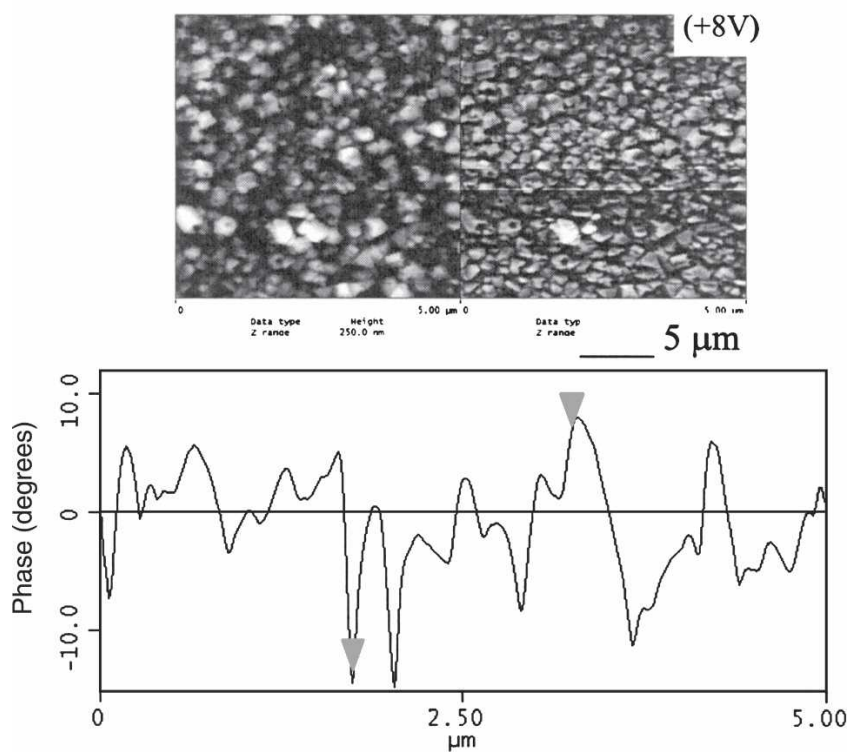

FIG. 7. (Continued)

shift $\phi$ between the driving force and the cantilever oscillation is given by ${ }^{56-58}$

$$
\tan (\phi)=-\frac{\gamma \omega}{\omega_{0}^{2}-\omega^{2}} .
$$

At resonance $\left(\omega=\omega_{0}\right)$, the phase shift is $-\pi / 2$. We adopt the standard convention of AFM that all measured phase shifts are $\Phi=\phi+\pi / 2$. Far from the substrate $(h \geqslant 100$ $\mathrm{nm})$, the phase lag is approximately 0 . If the cantilever is (c)

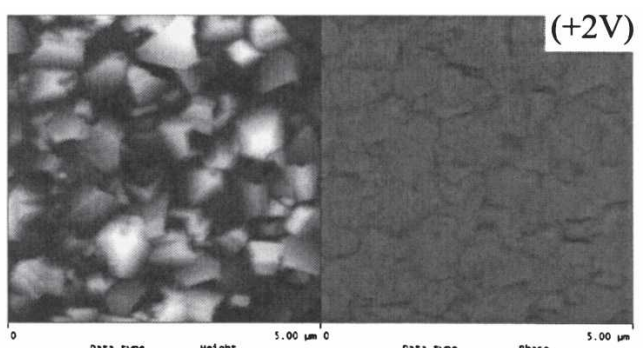

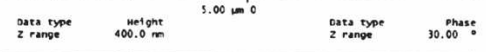
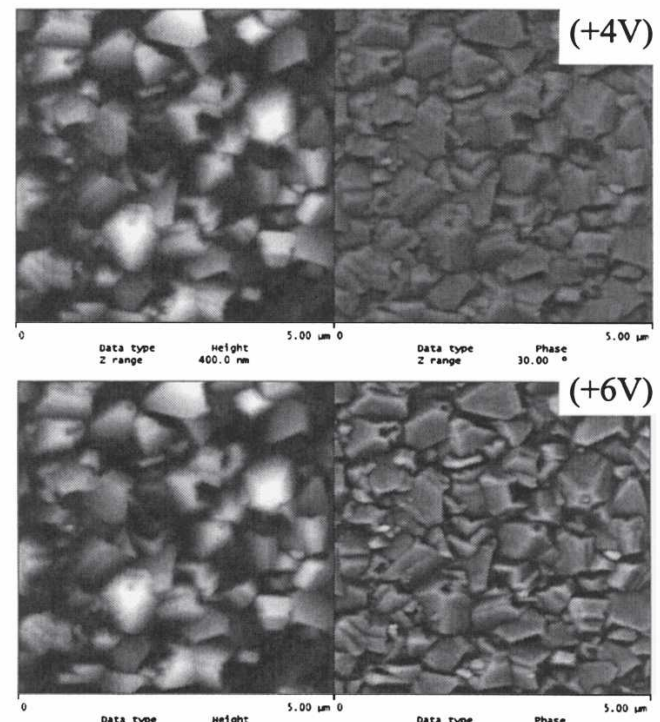

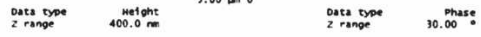
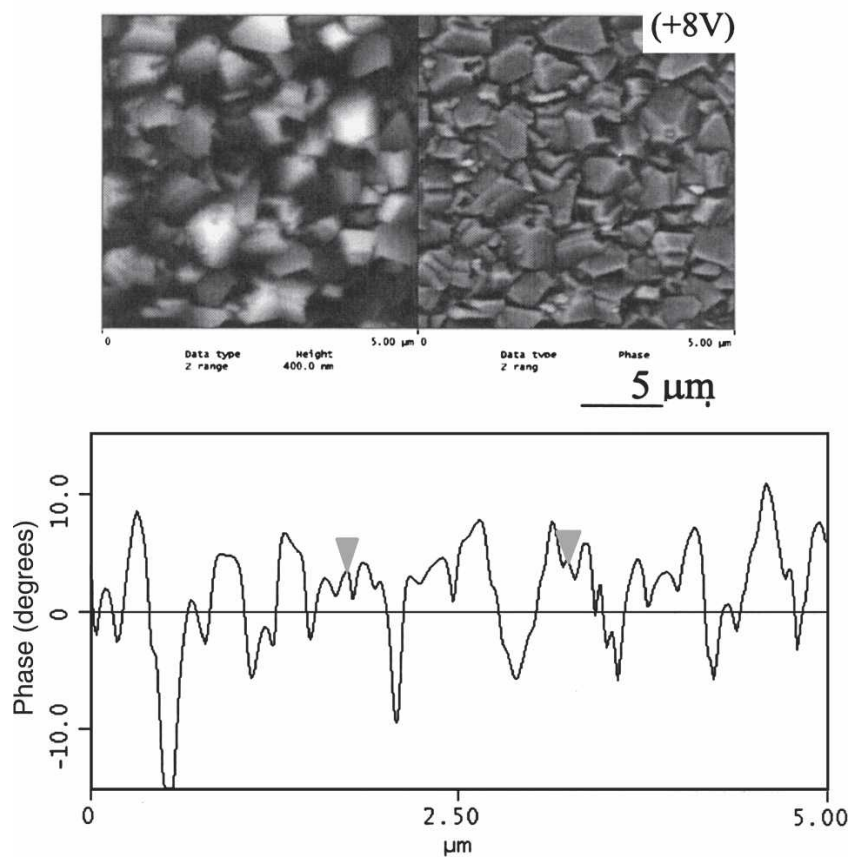

FIG. 7. (Continued)

scanned at height $h$ above a bare $\mathrm{SiO}_{2}$ substrate, the electrostatic force $F_{1}(h)$ between metallic tip and surface then changes the cantilever resonant frequency by $\Delta \omega$. For small $F_{1}(h)$, the frequency shift is proportional to the force gradient given by 
(d)

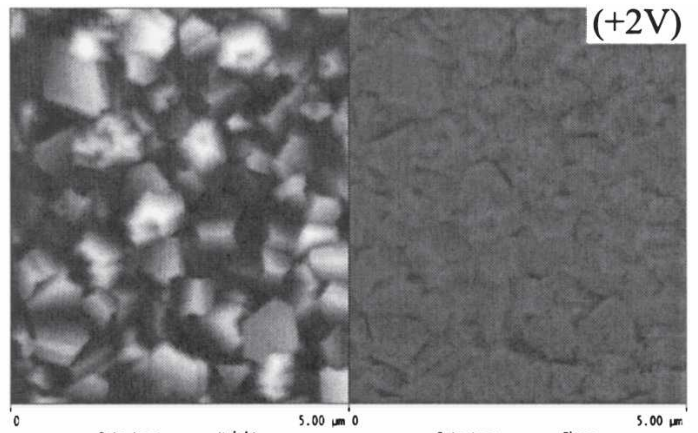

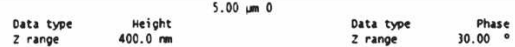

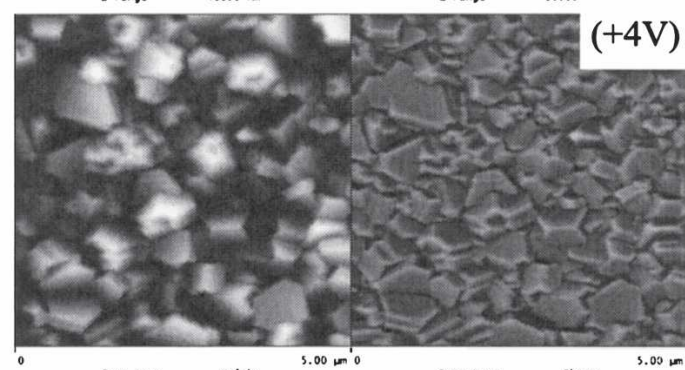

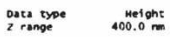

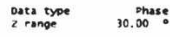

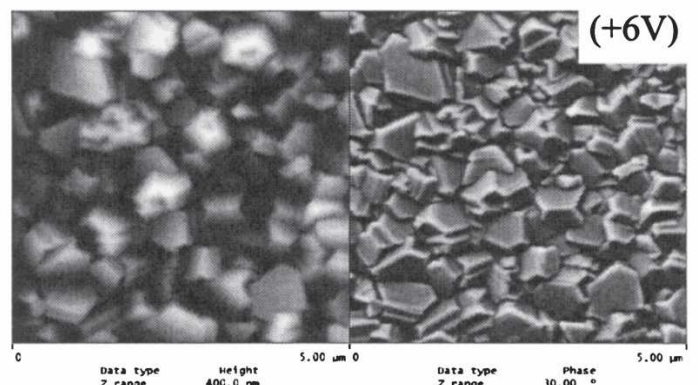

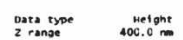

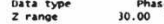
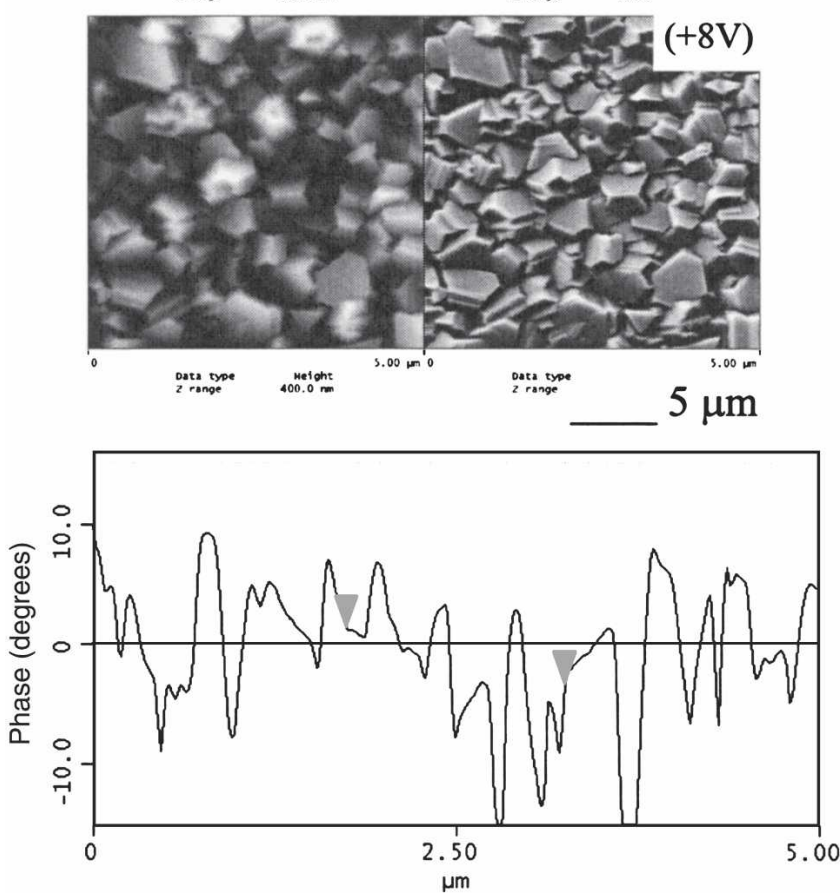

FIG. 7. (Continued)

$$
\Delta \omega \approx-\left(\omega_{0} / 2 k\right) F_{1}^{\prime}(h) \text {. }
$$

As a matter of fact, we found that the calculated frequency change is tens of $\mathrm{Hz}$ only. If $C_{1}(h)$ is the capacitance of the tip-substrate system, then

$$
F_{1}^{\prime}(h)=(1 / 2) C_{1}^{\prime}(h) V_{\text {tip }}^{2} \text {. }
$$

The attractive force $F_{1}(h)$ leads to a decrease of the resonant frequency of the cantilever (Fig. 1) and therefore to a negative phase value $\Phi_{0}$ for the background phase lag given by

$$
\tan \left(\Phi_{0}\right)=-\frac{Q}{2 k} C_{1}^{\prime \prime}(h) V_{\text {tip }}^{2},
$$

where $Q=\gamma \omega_{0}$ is the quality factor of the cantilever. This background value of the phase lag over the bare substrate is independent of the tip horizontal position and is used as the reference zero in Fig. 8. When the tip is above the sample to be probed, the total capacitance of the system is $C_{2}(h)$ with corresponding electrostatic force
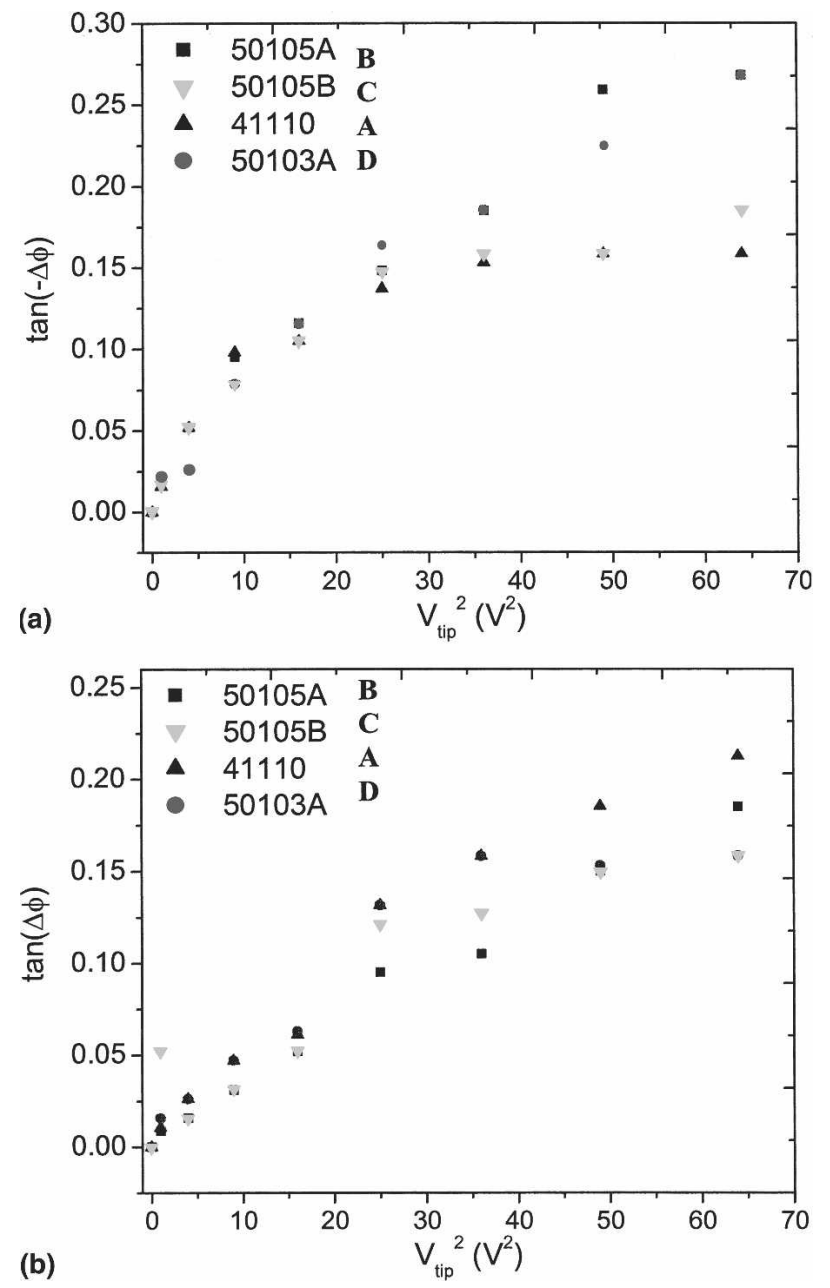

FIG. 8. Plot of measured absolute value of $\tan (\Delta \phi)$ versus $V_{\text {tip }}{ }^{2}$ for the heavily B-doped diamond films at different regions, evaluating both negative and positive phase shifts at a fixed scan height of $h=50 \mathrm{~nm}$. The graphs show a linear relation, as predicted by $\tan \left(\Phi-\Phi_{0}\right) \approx Q / 2 k$ $\left[C_{1}^{\prime \prime}(h)-C_{2}^{\prime \prime}(h)\right] V_{\text {tip }}^{2}$. 
$F_{2}^{\prime}(h)$. We note that the tip stays at the same height $h$ above the sample because it retraces the topography. Again, assuming that the electrostatic forces are small, the phase shift relative to that over the bare substrate is given by

$$
\tan \left(\Phi-\Phi_{0}\right) \approx \frac{Q}{2 k}\left[C_{1}^{\prime \prime}(h)-C_{2}^{\prime \prime}(h)\right] V_{\mathrm{tip}}^{2}
$$

Equations (7) and (8) predict that the tangents of both the phase background value and the phase shift above the BDD films vary linearly with tip voltage as $V_{\text {tip }}{ }^{2}$. Equation (8) also predicts that the sign of the phase shift in EFM is determined by the change in the second derivative of the total capacitance of the system. To explain the data quantitatively, we calculated the capacitance called for in Eqs. (7) and (8) using a simplified model for the geometry of the bare substrate and boron-doped diamond films. In each case, we approximate the tip with a circular parallel plate of radius $R_{\text {tip }}=30-40 \mathrm{~nm}$. Figure 8 displays the phase versus $V_{\text {tip }}{ }^{2}$ for all of the samples following the procedure mentioned above. All of the films show "positive-negative-positive" phase shifts when scanned over the surface, which will be accounted for in the following section.

For EFM over the bare substrate, the tip sits at a distance $h$ above the $\mathrm{SiO}_{2}$ dielectric layer with thickness $t=$ $200 \mathrm{~nm}$ on top of a ground plane [Fig. 9(a)]. Because the diameter of the crystallites in boron-doped diamond films are comparable to the tip radius $R_{\text {tip }}$, we model the BDD films as an insulating-semiconductive/conductive mixed phase plate of thickness $D$ and dielectric constant $\epsilon_{\mathrm{f}}$ at distances $h$ and $t$ from two conducting/ semiconducting planes [Fig. 9(b)]. Even these simple models of the sample geometry give very good agreement with the data, and accuracy could likely be improved through the use of more realistic geometries.

For tip-sample separation of $h=50 \mathrm{~nm}$, the tipsubstrate is modeled as a sphere above a dielectric plane rather than a metallic plate, and we demonstrate that within this range the predictions of the geometric models are in excellent quantitative agreement with the data. In the following, we restrict that a metallic plate is no longer appropriate, and the observed shifts are better described by modeling the tip-substrate system as a sphere above a dielectric plane, shown in Fig. 9. In the following, we restrict our observations to intermediate scan height of $h=50 \mathrm{~nm}$, and we show that within this range the predictions of the geometric models are in excellent quantitative agreement with the data. Over the bare substrate, the model presented in Fig. 9(a) predicts

$$
C_{1}^{\prime \prime}(h)=2 \epsilon_{0}\left(\pi R_{\mathrm{tip}}{ }^{2}\right) \frac{1}{\left(h+t / \epsilon_{S}\right)^{3}},
$$

where $\epsilon_{\mathrm{S}}=4.2$ is the $\mathrm{SiO}_{2}$ dielectric constant. ${ }^{19}$ For $h=50 \mathrm{~nm}$ and $t=200 \mathrm{~nm}$, we found $C_{1}^{\prime \prime}=48 \mu \mathrm{F} / \mathrm{m}^{2}$, while the experimental value from the slope of the line between phase and $V_{\text {tip }}{ }^{2}$ is $52 \mu \mathrm{F} / \mathrm{m}^{2}$. It seems that the measured values are in excellent agreement with the predictions.

We now turn our attention to the observed phase shifts over the BDD samples as a function of boron concentration and bias polarization shown in Fig. 7. For BDD samples, the geometry of Fig. 9(b) yields

$$
C_{2}^{\prime \prime}(h)=2 \epsilon_{0}\left(\pi R_{\text {tip }}{ }^{2}\right) \frac{1}{\left(h+t / \epsilon_{\mathrm{Si}}+D / \epsilon_{\mathrm{f}}\right)^{3}},
$$

where $\epsilon_{\mathrm{f}}$ is the BDD films' dielectric constant and $\epsilon_{\mathrm{Si}}$ is that of $p$-Si substrate. The Si surfaces were treated with diamond nanopowder colloidal solution, ultrasonicated, and rinsed, leaving behind particles sparsely which served as nucleating agents. Equations (7) and (8) predict that the phase shift for insulating regions of BDD films is always positive because $C_{2}^{\prime \prime}(h)<C_{1}^{\prime \prime}(h)$. The plot of both positive and negative phase shifts yields $C_{2}^{\prime \prime}(h)$. While the capacitance ranged between $28-67 \mathrm{pF}$, the dielectric constant is $7.8-8.4$, which seems slightly higher than
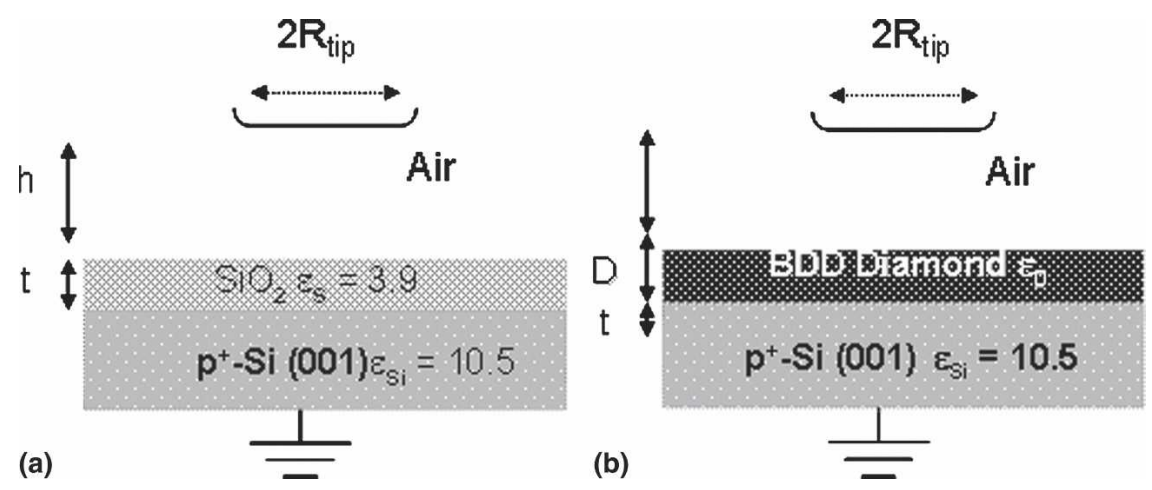

FIG. 9. (a, b) Model for the tip-sample geometry. The AFM tip (radius $R_{\text {tip }}=25 \mathrm{~nm}$ ) scans at a constant height $h$ above the sample. (b) The BDD sample is modeled as a dielectric plate of thickness $D=1 \mu \mathrm{m}(1000 \mathrm{~nm})$. These models are in excellent agreement with the experimental data for intermediate scan heights of $30-50 \mathrm{~nm}$. 
usual 5.5 for diamond at $79 \mathrm{kHz}$ in an ambient environment. ${ }^{59}$ This discrepancy was overcome by the fact that the films consist of mixed $s p^{3}$ - and $s p^{2}$-bonded carbon phases, and therefore the resulting value is an effective dielectric constant. Both the crystallite size and the extent of microstructural order also have important implications on these findings. These results are validated by SEM and Raman spectroscopy. For instance, with increasing boron concentration, the crystallite or grain size decreases and the Raman spectra confirmed a 16\% graphite impurity in the diamond nanoparticles as an upper bound. Figure 10 shows the relative dielectric constant as a function of the graphitic or $s p^{2}$-bonded carbon content. The increasing dielectric constant is attributed to the change in the crystal field caused by surface bond contraction of the micro-/nanocrystalline particles and to the conjugated double bonds or graphite phase with increasing boron concentration. ${ }^{60}$

Finally, we explain the negative-positive-negative (contrasting dark-bright-dark regions) phase shifts observed for BDD films. The fact that this contrast is seen for conducting samples, not for insulating material, rules out the possibility that it is an artifact of crosstalk from topography signal. To explain the negative phase shift, we note as the tip approaches the conducting region at height $h$, two forces act on the cantilever: (i) "capacitive force" from the tip-substrate interaction $F_{1}$ and (ii) an additional "attractive force" $F_{\text {tf }}$ due to the tip-substrate interaction. This additional force leads to a decrease in the phase $\Phi$, where $\tan \Phi=-(Q / k)\left(\mathrm{F}_{1}+\mathrm{F}_{\mathrm{tf}}^{\prime}\right)<$ $\tan \Phi_{0}=-(Q / k) F_{1}^{\prime}$, and thus to a negative phase shift $(\Delta \Phi)$. Although this model explains qualitatively the negative-positive-negative phase shift, but the numerical predictions are typically a factor of 1.5-2 times larger than the measured data. This suggests that the exact tipsample geometry should be taken into account to resolve this discrepancy to quantify the measurements.

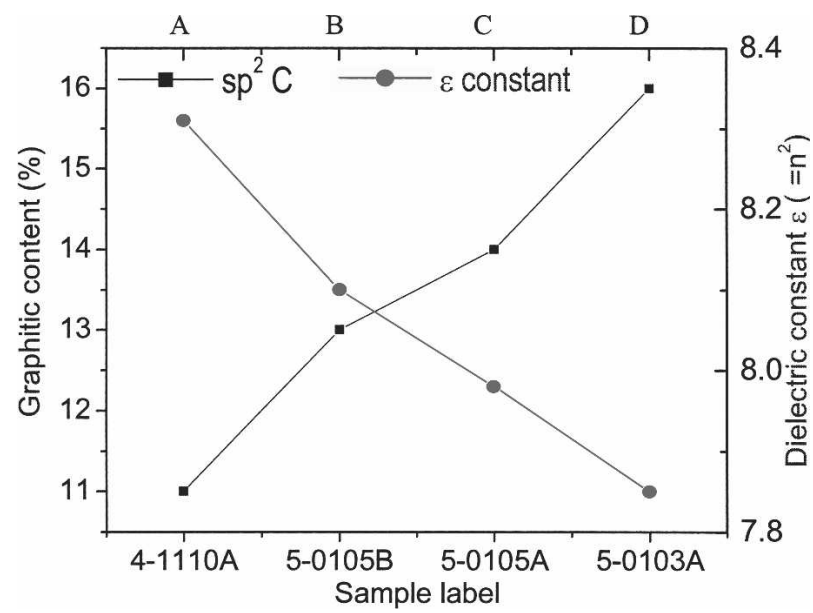

FIG. 10. Shown is the plot of variation of graphitic or nondiamond content $\left(s p^{2} \mathrm{C} \%\right)$ and relative dielectric constant $(\epsilon)$ for the heavily B-doped diamond films as a function of boron concentration.
In conclusion, the ". . . negative-positive-negative. . ." phase shift for the BDD films implies the existence of electrical conductivity inhomogeneity over the entire surface. Further, we introduced a quantitative model for phase shifts based on the change in the total capacitance of the tip-sample-substrate system. By using simple geometric models, we decoupled the topography signal from electrostatic force gradients and found quite an agreement with the data collected on BDD films. Finally, we have shown that the geometric model can be combined with the EFM-phase data to measure the dielectric constant of BDD films containing $s p^{3}$ - and $s p^{2}$-bonded carbon. Because this modeling is general, it can be used to determine the dielectric constant of other technological important materials in the context of electrical properties at micro-/nanoscale.

\section{CONCLUSION}

In summary, EFM-phase method was used to investigate the electrical conductivity heterogeneity of borondoped diamond films. The EFM-phase images showed that the films consisted of simultaneous topographic and nonelectrically active areas containing isolated sites of high conductivity and, thus, electrical activity. These sites were submicron in diameter and distinguished across the surface through sharp phase contrasts, i.e., darker and lighter regions. The distribution may be random across the surface, but it suggests that the conductive sites are at the grain boundaries and crystallite edges. The density of these sites increases with increasing boron doping level, which may be partly due the fact the decreasing crystallite size enhances the $s p^{2}$-bonded carbon. We also showed through analytical calculations by fitting and modeling through simple approximate geometries the experimental data well. These investigations show that the EFM-phase shift is related to the local surface potential, capacitance, and in-turn dielectric constant under various biases. It is likely that the micro-/ nanocrystalline BDD surface exhibits heterogeneity over the surface due to random distribution of dopant. These findings suggest that the area of high conductivity density correlates with a high boron doping level. The presence of a "positive-negative-positive-negative" phase shift along the line section indicates the presence of "insulating-conducting-insulating-conductive" phases, respectively, though qualitative. These findings were complemented and validated with SEM, XRD, and visible RS. It was found that decreasing grain size (alternatively increasing boron concentration) varies the dielectric constant, which is attributed to the change of the bond contraction of the nanosized particles. This pioneering study served to illustrate basic understandings of electrical properties at nanoscale through surface probe microscopy and how to engineer them via synthesis 
parameters. Moreover, some of the interesting findings highlight some of the important factors influencing electrical, field emission, and electrochemical properties for various applications, including metal recovery and oxidation of organic residues contained in industrial wastewaters.

\section{ACKNOWLEDGMENTS}

We gratefully acknowledge the facilities (SEM and AFM) housed in the Center for Applied Science and Engineering (CASE) at the Missouri State University, Springfield, MO, directed by Dr. R.E. Giedd and technical assistance by Mr. R.J. Patel, who helped in AFM and EFM measurements. This research work is supported by the summer faculty fellowship and internal grants awarded to the corresponding author (S. Gupta).

\section{REFERENCES}

1. J.A. Garrido, C.E. Nebel, M. Stutzmann, E. Gheeraert, N. Casanova, E. Bustarret, and A. Deneuville: A new acceptor state in CVD-diamond. Diamond Relat. Mater. 11, 347 (2002).

2. P.K. Bachmann and R. Messier: Diamond thin films. Chem. Eng. News 67, 24 (1989).

3. M.H. Nazare: Properties and Growth of Diamond, edited by G. Davies, EMIS Data Review Series (London, INSPEC, 1994), p. 85 .

4. P. John: The oxidation of (100) textured diamond. Diamond Relat. Mater. 11, 861 (2002).

5. A.E. Fischer, Y. Show, and G.M. Swain: Electrochemical performance of diamond thin-film electrodes from different commercial sources. Anal. Chem. 76, 2553 (2004).

6. M.A. Rodrigo, P.A. Michaud, I. Duo, M. Panizza, G. Cerisola, and Ch. Cominellis: Oxidation of 4-chlorophenol at boron-doped diamond electrode for wastewater treatment. J. Electrochem. Soc. 148, D60 (2001).

7. E. Kohn, P. Gluche, and M. Adamschik: Diamond MEMS — a new emerging technology. Diamond Relat. Mater. 8, 934 (1999).

8. S. Gupta, B.L. Weiss, B.R. Weiner, L. Pilione, A. Badzian, and G. Morell: Electron field emission properties of $\gamma$-irradiated microcrystalline diamond and nanocrystalline carbon thin films. J. Appl. Phvs. 92, 3311 (2002).

9. A.V. Sumant, D.S. Grierson, J.E. Gerbi, J. Birrell, U.D. Lanke, O. Auciello, J.A. Carlisle, and R.W. Carpick: Toward the ultimate tribological interface: Surface chemistry and nanotribology of ultrananocrystalline diamond. Adv. Mater. 17, 1039 (2005).

10. J.B. Cui, J. Robertson, and W.I. Milne: The effect of film resistance on electron field emission from amorphous carbon films. Diamond Relat. Mater. 10, 868 (2001).

11. K.H. Chen, Y.L. Lai, L.C. Chen, J.Y. Wu, and F.J. Kao: Hightemperature Raman study in CVD diamond. Thin Solid Films $\mathbf{2 7 0}$. 143 (1995).

12. W.A. Yarbrough and R. Messier: Current issues and problems in the chemical vapor deposition of diamond. Science 247, 688 (1990).

13. R. Kalish: The search for donors in diamond. Diamond Relat. Mater. 10, 1749 (2001).

14. A.W.S. Williams, E.C. Lightowlers, and A.T. Collins: Impurity conduction in synthetic semiconducting diamond. J.Phys. C: Solid State Phys. 3, 1727 (1970).
15. E. Bustarret, E. Gheeraert, and K. Watanabe: Optical and electronic properties of heavily boron-doped homo-epitaxial diamond. Phys. Status Solidi 199, 9 (2004).

16. R. Viswanathan and M.B. Heaney: Direct imaging of the percolation network in a three-dimensional disordered conductor-insulator composite. Phvs. Rev. Lett. 75, 4433 (1995).

17. K.B. Holt, A.J. Bard, Y. Show, and G.M. Swain: Scanning electrochemical microscopy and conductive probe atomic force microscopy studies of hydrogen-terminated boron-doped diamond electrodes with different doping levels. J.Phys. Chem. B 108. 15117 (2004).

18. J. Heo and M. Bockrath: Local electronic structure of singlewalled carbon nanotubes from electrostatic force microscopy. Nano Lett. 5, 853 (2005).

19. C. Stali, T. Johnson, Jr., and N.J. Pinto: Quantitative analysis of scanning conductance microscopy. Nano Lett. 4, 859 (2004).

20. S. Jeffery, A. Oral, and J.B. Pethica: Quantitative electrostatic force measurement in AFM. Appl. Surf. Sci. 157, 280 (2000).

21. M. Bockrath, N. Markovic, A. Shepard, M. Tinkham, L. Gurevich, L.P. Kouwenhoven, M.W. Wu, and L.L. Sohn: Scanned conductance microscopy of carbon nanotubes and $\lambda$-DNA. Nano Lett. 2. 187 (2002).

22. O. Enea, B. Riedo, and G. Dietler: AFM study of Pt clusters electrochemically deposited onto boron-doped diamond films. Nano Lett. 2, 241 (2002).

23. C.H. Lei, A. Das, M. Elliott, and J.E. MacDonald: Quantitative electrostatic force microscopy-phase measurements. J. Nanotechnol. 15, 627 (2004).

24. H. Sakaue, N. Yoshimura, S. Shingubara, and T. Takahagi: Lowdielectric constant porous diamond films formed by diamond nanoparticles. Appl. Phvs. Lett. 83, 2226 (2003).

25. O.A. Williams, M. Daenena, J. D'Haen, K. Haenen, J. Maes, V.V. Moshchalkov, M. Nesládek, and D.M. Gruen: Comparison of the growth and properties of ultrananocrystalline diamond and nanocrystalline diamond. Diamond Relat. Mater. 15, 230 (2005).

26. O.A. Williams, S. Curat, R.B. Jackman, J.E. Gerbi, and D.M. Gruen: $n$-Type conductivity in ultrananocrystalline diamond films. Appl. Phvs. Lett. 85, 1680 (2004).

27. P.R. Gill, W. Murray, and M.H. Wright: Practical optimization, in The Levenberg-Marquardt Method (Academic Press, London, 1981), Sec. 4.7.3, pp. 136-137.

28. D.M. Gruen: Nnaocrystalline diamond. Annu. Rev. Mater. Sci. 29. 211 (1999).

29. S. Gupta, B.R. Weiner, and G. Morell: Synthesis and characterization of sulfur-incorporated microcrystalline diamond and nanocrystalline carbon thin films by hot filament chemical vapor deposition. J. Mater. Res. 18, 363 (2003).

30. B.D. Cullity: Elements of X-ray Diffraction, 2nd Ed. (AddisonWesley, Reading, MA, 1978), pp. 102-111.

31. M. Yoshikawa, Y. Mori, H. Obata, M. Maegawa, G. Katagiri, H. Ishida, and A. Ishitani: Raman scattering from nanometer-sized diamond. Appl. Phvs. Lett. 67, 694 (1995).

32. S. Prawer and R.J. Nemanich: Raman spectroscopy of diamond and doped diamond. Philos. Trans. R. Soc. London, Ser. A 13, 2537 (2004).

33. A.E. Fischer, Y. Show, and G.M. Swain: Electrochemical performance of diamond thin-film electrodes from different commercial sources. Anal. Chem. 76, 2553 (2004).

34. J.A. Bennett, J. Wang, Y. Show, and G.M. Swain: Effect of $s p^{2}$ bonded nondiamond carbon impurity on the response of borondoped polycrystalline diamond thin-film electrodes. chem. Soc. 151, E306 (2004).

35. M. Mermoux, B. Marcus, G.M. Swain, and J.E. Butler: A confocal Raman imaging study of an optically transparent boron-doped diamond electrode. J.Phvs. Chem. B 106, 10816 (2002). 
36. R.J. Nemanich, J.T. Glass, G. Lucovsky, and R.E. Shroder: Raman scattering characterization of carbon bonding in diamond and diamondlike thin films. J. Vac. Sci. Technol., A 6, 1783 (1988).

37. D.S. Knight and W.B. White: Characterization of diamond films by Raman spectroscopy. J. Mater. Res. 4, 385 (1989).

38. L. Bergman and R.J. Nemanich: Raman and photoluminescence analysis of stress state and impurity distribution in diamond thin films. J. Appl. Phvs. 78, 6709 (1995).

39. P. Gonon, E. Gheeraert, A. Deneuville, F. Fontaine, L. Abello, and G. Lucazeau: Characterization of heavily B-doped polycrystalline diamond films using Raman spectroscopy and electron spin resonance. J. Appl. Phvs. 78, 7059 (1995).

40. J.W. Ager, III, W. Walukiewicz, M. McMluskey, M.A. Plano, and M.I. Landstrass: Fano interference of the Raman phonon in heavily boron-doped diamond films grown by chemical vapor deposition. Appl. Phvs. Lett. 66, 616 (1995).

41. K. Ushizawa, K. Watanabe, T. Ando, I. Sakaguchi, M. NishitaniGamo, Y. Sato, and H. Kanda: Boron concentration dependence of Raman spectra on $\{100\}$ and $\{111\}$ facets of B-doped CVD diamond. Diamond Relat. Mater. 7, 1719 (1998).

42. P. Pruvost, E. Bustarret, and A. Deneuville: Characteristics of homoepitaxial heavily boron-doped diamond films from their Raman spectra. Diamond Relat. Mater. 9, 295 (2000).

43. P. Pruvost and A. Deneuville: Analysis of the Fano in diamond. Diamond Relat. Mater. 10, 531 (2001).

44. S. Prawer, K.W. Nugent, D.N. Jamieson, J.O. Orwa, L.A. Bursill, and J.L. Peng: The Raman spectrum of nanocrystalline diamond. Chem. Phvs. Lett. 332, 93 (2000).

45. A.C. Ferrari and J. Robertson: Origin of the $1150-\mathrm{cm}^{-1}$ Raman mode in nanocrystalline diamond. Phvs. Rev. B 63, 121405 (2001).

46. A.C. Ferrari and J. Robertson: Interpretation of Raman spectra of disordered and amorphous carbon. Phvs. Rev. B 61, 14095 (2000).

47. H. Kuzmany, R. Pfeiffer, N. Salk, and B. Günther: The mystery of the $1140 \mathrm{~cm}^{-1}$ Raman line in nanocrystalline diamond films. Carbon 42, 911 (2004).

48. D.M. Gruen, M.A. Krauss, R. Zuiker, C.D. Csencsits, R. Terminello,
J.A. Carlisle, I. Jimenez, D.G.J. Sutherland, D.K. Shu, W. Tong, and F. Himpsel: Characterization of nanocrystalline diamond films by core-level photoabsorption. Appl. Phvs. Lett. 68, 1640 $\underline{(1996)}$.

49. J.F. Bresse and S. Blayac: Epitaxial layer sheet resistance outside and under ohmic contacts measurements using electrostatic force microscopy. Solid State Electron. 45, 1071 (2001).

50. J.F. Bresse: Contact potential difference of $\mathrm{Au}$ and GaInAs by electrostatic force microscopy. Mikrochim. Acta 132, 449 (2000).

51. J.C. Angus and C.C. Hayman: Low-pressure, metastable growth of diamond and "diamondlike" phases. Science 241, 913 (1988).

52. A. Argoitia, J.C. Angus, J.S. Ma, L. Wang, P. Pirouz, and W.R.L. Lambrecht: Heteroepitaxy of diamond on c-BN: growth mechanisms and defect characterization. J.Mater. Res. 9, 1849 (1994).

53. M.I. Landstrass and K.V. Ravi: Hydrogen passivation of electrically active defects in diamond. Appl. Phvs. Lett. 55, 1391 (1989).

54. T. Kolber, K. Piplits, R. Haubner, and H. Hutter: Quantitative investigation of boron incorporation in polycrystalline CVD diamond films by SIMS. Fresenius J. Anal. Chem. 365, 636 (1999).

55. A.A. Middleton and N.S. Wingreen: Collective transport in arrays of small metallic dots. Phvs. Rev. Lett. 71, 3198 (1993).

56. R. Weisendanger: Scanning Probe Microscopy and Spectroscopy: Methods and Applications (Cambridge University Press, Cambridge, England, 1994).

57. D.A. Bonnell and B.L. Huey: Scanning Probe Microscopy and Spectroscopy: Theory, Techniques and Applications, 2nd ed. (John Wiley \& Sons, New York, 2001), pp. 8-42.

58. S.V. Kalinin and D.A. Bonnell: Scanning Probe Microscopy and Spectroscopy: Theory, Techniques and Applications, 2nd ed. (John Wiley \& Sons: New York, 2001), pp. 205-251.

59. H. Ye, H. Yan, and R.B. Jackman: Dielectric properties of single crystal diamond. Semicond. Sci. Technol. 20, 296 (2005).

60. H. Ye, C.Q. Sun, and P. Hing: Control of grain size and size effect on the dielectric constant of diamond films. J.Phvs. D: Appl. Phvs. 33, L148 (2000). 